\title{
Microglia are Involved in Pruritus Induced by DNFB via the CX3CR1/p38 MAPK Pathway
}

\author{
Ying Zhang Jia Yan Rong Hu Yu Sun Yiwen Ma Zhifeng Chen Hong Jiang \\ Department of Anesthesiology, Shanghai Ninth People's Hospital Affiliated to Shanghai Jiao Tong \\ University, School of Medicine, Shanghai, China
}

\section{Key Words}

Microglia $•$ Pruritus $・$ DNFB $・$ CX3CR1/p38 MAPK pathway

\begin{abstract}
Background/Aims: Pruritus, also known as itch, is a common, unpleasant sensation that can be difficult to treat. Frequently, chronic itch is associated with the development of neuropathic pain resulting from nerve injury or insult. Previous studies have shown the involvement of spinal microglia in the development of neuropathic pain, but their role in chronic pruritus is unclear. Methods: For this study, we constructed a model of chronic pruritus in mice using repeated applications of 2, 4-dinitrofluorobenzene (DNFB) and showed prolonged scratching behavior in treated mice that continued for at least $7 \mathrm{~d}$ after the final DNFB treatment. Results: Scratching was accompanied by activation of spinal microglia and both were reduced by an inhibitor of microglial activity. We also showed that microglial activation entailed increased signaling in the p38 MAPK pathway, and treatment with a p38 inhibitor reduced scratching in DNFBtreated mice. We also examined the role of fractalkine/CX3CR1 signaling in the development of DNFB-induced pruritus and showed that intrathecal administration of antiserum against either CX3CR1or FKN inhibited p38 activity and decreased scratching. Conclusion: Our results suggest that microglia are involved in pruritus induced by DNFB via FKN/CX3CR1/p38MAPK pathways similar to those participating in the development of neuropathic pain.
\end{abstract}




\section{Introduction}

Pruritus, also known as itch, is a common, sometimes unpleasant sensation that elicits an urge to scratch [1]. Itch can be categorized as either acute or chronic, depending on its persistence [2]. Acute itch acts as an alarm mechanism to prevent bodily injury by provoking the scratch reflex to remove noxious external stimuli from the skin [3]. Chronic itch, persisting for more than six weeks, may be a symptom of more serious dermatological or systemic diseases and can have a detrimental effect on an individual's quality of life. Neuropathic itch syndromes, in which the itch sensation appears out of proportion or independent of pruritogenic stimuli [4], are chronic conditions that result from peripheral or central nervous system dysfunction [5] and may potentially involve neuronal or glial damage[6, 7]. Acute pruritus is mediated primarily by histamine-sensitive neurons in the skin [8] and may be partially alleviated by antihistamines or topical steroids [4]. The mechanisms underlying neuropathic pruritus, in contrast, have not been characterized and there are no effective treatments for this condition short of behavioral interventions and localized anesthetics to reduce the scratch reflex [5]. A more thorough understanding of these mechanisms is urgently required to provide for more effective treatments.

Pain and itch are major somatosensory sensations both conveyed by primary sensory neurons to the dorsal root ganglion and processed there by second-order neurons for transmittal to supraspinal locations where individual perceptions are formed $[9,10]$. Pruritus is strongly associated with chronic pain [11], and in some cases of atopic dermatitis normally painful stimuli can result in the inappropriate sensation of itch [12]. Spinal interneurons can inhibit itch pathways and may serve as mediators between pain and pruritic signaling [13, 14]. Pain and pruritic signaling systems may utilize common receptors including toll-like receptors [15]. Diffuse activation maps for itch in functional brain studies appear to overlap, although they are not identical to pain maps $[16,17]$. Furthermore, itch sensation can be suppressed by activation of pain neurons [18] while blockade of nociception may result in the urge to itch [19].

Central to the intertwining of pain and itch pathways are microglia, resident innate immune cells originating from primitive myeloid precursors [20]. Increasing evidence suggests that microglial activity plays a role in the pathogenesis of neuropathic pain. Nerve injury can induce expression of microglial-specific markers [21] and increase production of proinflammatory cytokines that may facilitate neuropathic pain [15, 22] Expression of the microglial-specific protein ionized calcium binding adaptor molecule 1 (Iba1) is upregulated in activated microglia of the facial nucleus following facial nerve axotomy [23]. Inhibition of spinal glia can block or reduce neuropathic pain [24]. Members of the mitogenactivated protein kinase (MAPK) family, including extracellular signal-regulated kinase (ERK) and p38 MAPK, play key roles in the activation of microglia following nerve injury. ERK is phosphorylated in dorsal horn neurons as early as 10 min following spinal nerve ligation, followed by widespread induction in spinal microglial cells [25]. p38 is activated by phosphorylation in spinal microglia shortly after nerve injury and peaks $3 \mathrm{~d}$ later [26]. minocycline, a specific inhibitor of microglial activation [27], has been shown to inhibit neuropathic pain $[28,29]$ by decreasing levels of phospho-p38 (p-p38). ERK phosphorylation has been demonstrated in cases of histamine-dependent acute itch as well as in chronic pruritus that developed after repeated administration of 2,4-dinitrofluorobenzene (DNFB) to the skin of mice [30]. However, the role of p38 in the development of chronic pruritus has not been fully elucidated.

Chemokine receptors in microglial cells, CCR2 and CX3CR1, as well as the ATP receptor P2X4, are upregulated following nerve injury [31-33]. Neutralizing antibodies against CX3CR1 reduced mechanical allodynia following spinal nerve ligation and blocked activation of p38 in spinal microglia [33]. Fractalkine (FKN), also known as CX3CL1, is the sole ligand for the CX3CR1 receptor and is a potential mediator of neural/glial interactions [34] FKN is produced by dorsal root ganglion neurons [32], activates microglial cells following spinal nerve ligation [33] and produces allodynia and hyperalgesia [35]. In addition, intrathecal 
administration of FKN strongly induced p38 phosphorylation/activation in spinal microglial cells [33]. To our knowledge, FKN/CX3CR1 signaling has not been demonstrated in models of chronic pruritus.

The goal of our research was to elucidate mechanisms involved in chronic pruritus using a previously-described mouse model of allergic dermatitis [36]. Repeated application of DNFB to nape skin produces dermatitis, elevated serum immunoglobulin E and provokes vigorous scratching behavior. Our results showed that activation of spinal cord microglia was involved in the development of pruritus utilizing the same FKN/CX3CR1/p38MAPK signaling pathways which also mediate the development of neuropathic pain.

\section{Materials and Methods}

Animals

Male adult ICR mice (25-35g) purchased from the Shanghai Laboratory Animal Center (Shanghai, China) were used. Experiments were performed in accordance with the National Institutes of Health Guidelines for the Care and Use of Laboratory Animals. Animal care procedures were reviewed and approved by the Animal Study Committee at Jiaotong University School of Medicine, Shanghai, China. Animals were housed under a $12 \mathrm{~h}$ light/dark cycle in a pathogen-free area with free access to water and food.

Drugs and administration

To induce chronic pruritus, $150 \mu \mathrm{l}$ of $0.15 \%$ DNFB (Sigma-Aldrich, St. Louis, MO) in acetone was painted onto the shaved skin of the mouse nape for a total of four applications, twice in the first week and once per week for the next two weeks. Control mice received the same number of treatments, but were painted with acetone alone.

To study the effect of minocycline on pruritus induced by DNFB, 30mg/kg of minocycline (SigmaAldrich) was dissolved in sterile saline and administered via intrathecal injection 20min after the first DNFB painting, then once daily until the mice were sacrificed. The control groups received a vehicle (sterile saline for injection) according to the same schedule.

To study the role of p38, CX3CR1and FKN in the development of chronic pruritus, we performed blocking studies using the p38 inhibitor SB239063 (Sigma-Aldrich), the neutralizing antibody anti-mouse CX3CR1(Abcam, Cambridge, UK), and rabbit polyclonal anti-FKN (Abcam), respectively. Reagents were injected intrathecally $20 \mathrm{~min}$ after the first DNFB painting, then once daily until the mice were sacrificed.

Behavioral analysis

Itch response evaluation was performed as described previously[37]. Hind paw scratching behaviors directed towards the painting site were recorded for 30 min starting at $2 \mathrm{~h}, 3 \mathrm{~d}$ and $7 \mathrm{~d}$ after the last application of DNFB. For some experiments, the scratching behavior was recorded only on the third day following the last DNFB painting.

\section{Immunohistochemistry}

Mice were deeply anesthetized with $10 \%$ chloralhydrate and perfused through the ascending aorta with PBS followed by $4 \%$ paraformaldehyde in $0.1 \mathrm{M}$ phosphate buffer $(\mathrm{pH} 7.4)$. After the perfusion, the spinal cord and nape skin were removed and post-fixed in $4 \%$ paraformaldehyde in $0.1 \mathrm{M}$ phosphate buffer (pH 7.4) overnight. Samples were cut transversely into $30 \mu \mathrm{m}$-thick frozen sections on a cryostat. Six section was collected as one set per animal and processed for immunofluorescence.

The following primary antibodies were used: goat polyclonal anti-Iba1 (1:500, Santa Cruz Biotechnology, Santa Cruz, CA), rabbit polyclonal anti-phospho-p38 MAPK(1:500, Cell Signaling Technology, Danvers, MA), rabbit polyclonal anti-p38 MAPK(1:500, Cell Signaling Technology, Danvers, MA), rabbit polyclonal anti-CX3CR1 (1:1000, Abcam), and rabbit polyclonal anti-FKN (1:1000, Abcam). Sections were incubated with primary antibodies overnight at $4^{\circ} \mathrm{C}$, and then with Alexa Fluor 488/594-conjugated species-specific secondary antibodies (1:1000; Invitrogen, Carlsbad, CA) for $1 \mathrm{~h}$ at room temperature. In some experiments, double immunofluorescence staining was performed in which sections were incubated with a mixture of rabbit polyclonal and goat polyclonal primary antibodies, followed by a mixture of Alexa Fluor 488 and Alexa Fluor 594 secondary antibodies.

\section{KARGER}


Fig. 1. Microglia in the spinal cord were activated 3 days after DNFB treatment. (A)Bar graph showing scratching behavior in mice treated 4 times by DNFB painting with and without intrathecal administration of minocycline. Hind paw scratches were recorded for $30 \min 2 \mathrm{~h}, 3 \mathrm{~d}$ and $7 \mathrm{~d}$ after the final DNFB painting. ${ }^{* * *} \mathrm{P}<0.001 \mathrm{v}$. DNFB treatment alone; bars represent mean \pm SEM; $n=6$ for each group. (B)Representative images of cervical spinal cord sections immunostained with an antibody against Iba1show activated microglia 3 dand $7 \mathrm{~d}$ after DNFB treatment and the effect of minocycline administration on microglial activation $3 \mathrm{~d}$ after DNFB. Scale bar equals $100 \mu \mathrm{m}$.

Western blots

To examine the change in p-p38 protein expression, we performed western blots using spinal cord protein extracts from the experimental groups of mice. Cervical cord segments were dissected after behavioral tests and homogenized in T-PER Tissue Protein Extraction Reagent with $10 \% \mathrm{v} / \mathrm{v}$ Halt $^{\mathrm{TM}}$ proteinase and phosphatase inhibitorcocktail (ThermoFisher Scientific, Waltham, MA). After centrifugation at $12000 \mathrm{rpm}$ for $15 \mathrm{~min}$ at $4^{\circ} \mathrm{C}$, the supernatant was collected, boiled with protein loading buffer at $100^{\circ} \mathrm{C}$ for 10 min and subjected to SDS-PAGE electrophoresis. Proteins were transferred to cellulose nitrate membranes. Blots were incubated with p38, p-p38 or CX3CR1 primary antibodies (1:1000 dilution for each) or mouse monoclonal anti-beta actin (1:5000, Abcam) overnight at $4^{\circ} \mathrm{C}$, followed by horseradish

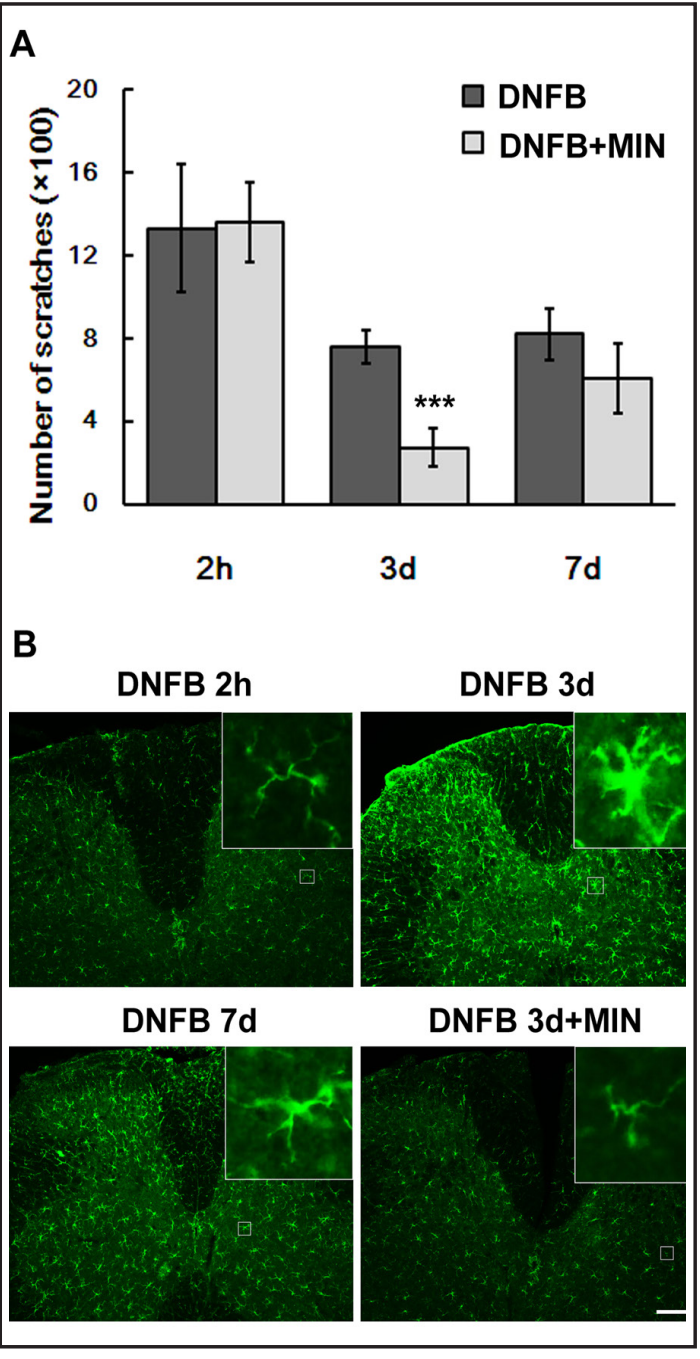
peroxidase-conjugated secondary antibodyfor $1 \mathrm{~h}$ at room temperature, then visualized with the Luminata ${ }^{\mathrm{TM}}$ Crescendo western HRP substrate (Millipore, Temecula, CA) .

\section{Quantification and statistics}

All data are presented as means \pm SEM. Differences between groups were compared using Student's t-tests. P values $<0.05$ were considered significant.

\section{Results}

Microglia were activated 3 days after DNFB treatment

DNFB treatment was previously established as a model of contact dermatitis [36] that has been utilized to produce acute itch [30,38] and allergic pruritus [39] in mice. We recorded scratching behavior for $30 \mathrm{~min}$ at progressive time points following the cessation of DNFB treatment in mice receiving DNFB painting with and without intrathecal administration of the microglial inhibitor minocycline (Fig. 1A). Scratching behavior was vigorous $2 \mathrm{~h}$ after the last DNFB treatment and, despite some subsidence in frequency, was still prevalent in DNFB-treated mice $7 \mathrm{~d}$ after the last painting, demonstrating the chronic nature of pruritus produced in this model. minocycline administration had no effect on scratching behavior 2 $\mathrm{h}$ after the final DNFB painting, but significantly reduced scratching by more than $50 \% 3 \mathrm{~d}$ after the last painting, compared to mice treated with DNFB alone. The effect of minocycline was no longer active $7 \mathrm{~d}$ after the last painting, when scratching was similar in both groups. 


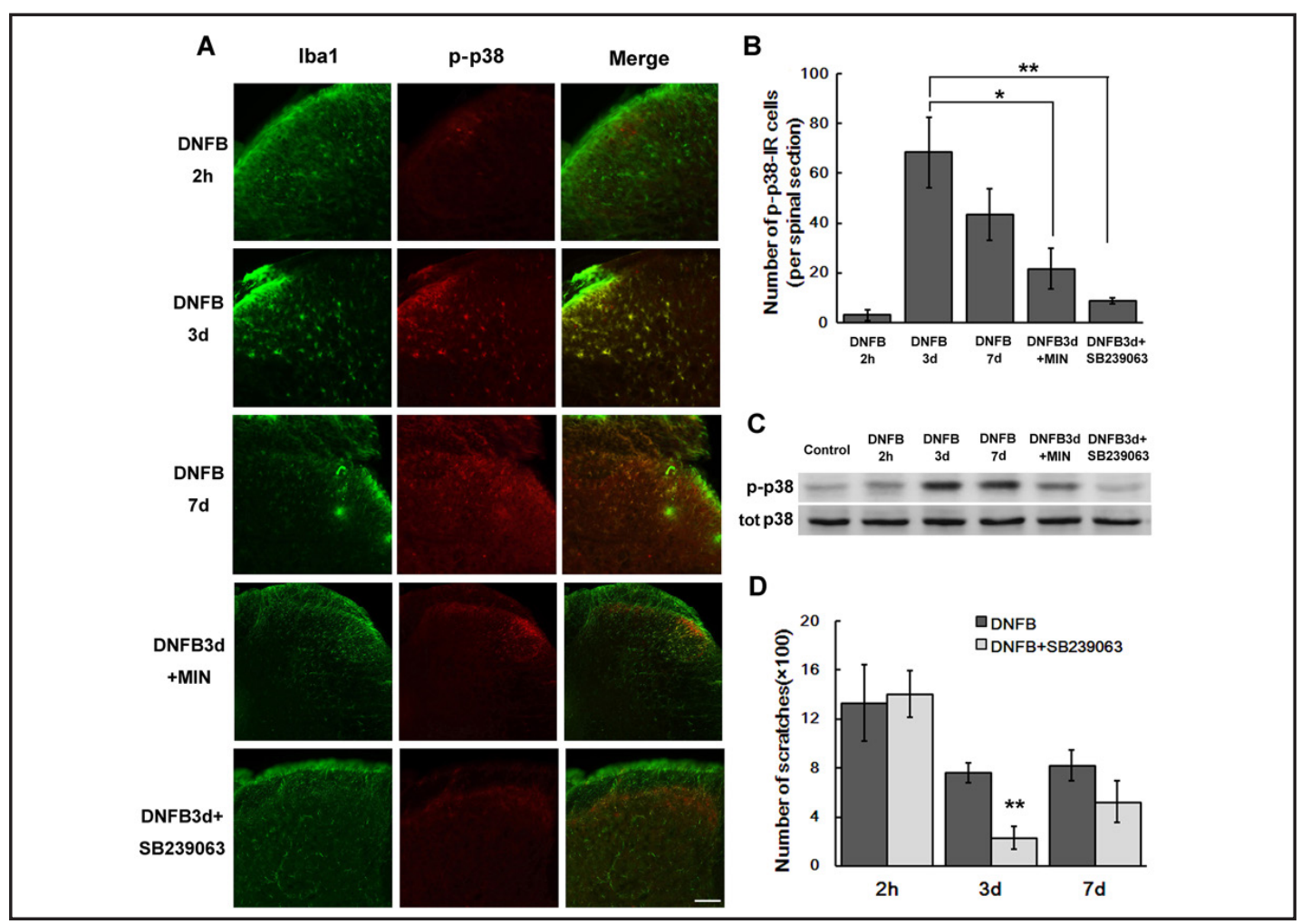

Fig. 2. p38 MAPK pathway in microglia was activated 3 days after DNFB treatment. (A) Representative images of cervical spinal cord sections immunostained with anti-Iba1 and anti-p-p38 antisera $2 \mathrm{~h}, 3 \mathrm{~d}$ and 7 $\mathrm{d}$ after the end of DNFB treatment; additional animals received concomitant treatment with minocycline or SB239063 and were analyzed 3 d following DNFB treatment.Scale bar equals $100 \mu \mathrm{m}$.(B) Bar graph showing the number of p-p38 immunoreactive (IR) cells per spinal cord section from animals with treatments indicated in $(\mathrm{A}) .{ }^{* *} \mathrm{P}<0.01$; bars represent mean $\pm \mathrm{SEM}$; $\mathrm{n}=6$ for each group; Six sections per animal were selected for counting. (C) Representative western blot of p-p38 and total p38 expression in spinal cord extracts from control and DNFB-treated animals $2 \mathrm{~h}, 3 \mathrm{~d}$ and $7 \mathrm{~d}$ after the end of treatment; additional animals received intrathecal minocycline or SB239063 and were analyzed $3 \mathrm{~d}$ after the last DNFB administration. (D)Bar graph showing the number of hind paw scratches in $30 \mathrm{~min}$ from animals receiving DNFB alone or DNFB with intrathecal administration of SB239064 recorded at $2 \mathrm{~h}, 3 \mathrm{~d}$ and $7 \mathrm{~d}$ after the cessation of DNFB painting. ${ }^{* *} \mathrm{P}<0.01 \mathrm{v}$. DNFB treatment alone; bars represent mean $\pm \mathrm{SEM} ; \mathrm{n}=6$ for each group.

Since minocycline can inhibit microglial activation, we examined if intrathecal administration of minocycline affected microglial activation as shown by the microglialspecific marker Iba1 [23]. Results showed that microglial activation in the spinal cord increased $3 \mathrm{~d}$ after the cessation of DNFB treatment and that activation continued to a least the $7 \mathrm{~d}$ time point (Fig. 1B). However, microglial activation did not occur until after the onset of scratching behavior, which was vigorous $2 \mathrm{~h}$ after the last DNFB painting. minocycline treatment reduced the number and intensity of Iba1-stained microglial cells at the $3 \mathrm{~d}$ time point, suggesting that reduced microglial activation was associated with diminished scratching at this time point.

\section{p38 MAPK pathway in microglia was activated 3 days after DNFB treatment}

To examine the possible involvement of the p38 MAPK pathway in the response of microglial cells to pruritogenic stimuli, we immunostained spinal cord sections for Iba1 and p-p38 expression 2 h, $3 \mathrm{~d}$ and $7 \mathrm{~d}$ after the last DNFB treatment (Fig. 2A). Separate groups of animals received intrathecal treatments with minocycline or the p38 inhibitor SB239063 during DNFB painting. Iba1 labeling in the spinal cord was increased $3 \mathrm{~d}$ and $7 \mathrm{~d}$ after the 
Fig. 3. CX3CR1 expression in microglia was induced by DNFB treatment. (A)Representative western blot showing CX3CR1 expression in spinal cord extracts $3 \mathrm{~d}$ after final DNFB treatment in animals treated with DNFB alone and in animals receiving concomitant intrathecal administration of minocycline or SB239063 compared to acetone-painted control animals. $\beta$-actin expression served as a loading control. (B)Representative confocal imagesof cervical spinal cord sections showing CX3CR1 and Iba1 immunostaining3 dafter final DNFB treatment in animals from treatment groups described in $3 \mathrm{~A}$. $n=6$ for each group;scale bar equals $50 \mu \mathrm{m}$.

end of treatment and correlated with an increase in the extent and intensity of p-p38 staining. Quantitation of the number of p-p38-stained cells per section showed an approximately 14fold and 8-fold increase in the number of immunoreactive cells at the $3 \mathrm{~d}$ and 7d time points, respectively (Fig. 2B). Minocycline and SB239063 treatment significantly reduce the number of p-p38-labeled cells $3 \mathrm{~d}$ after the end of DNFB painting compared to non-treated mice at the same time point, showing

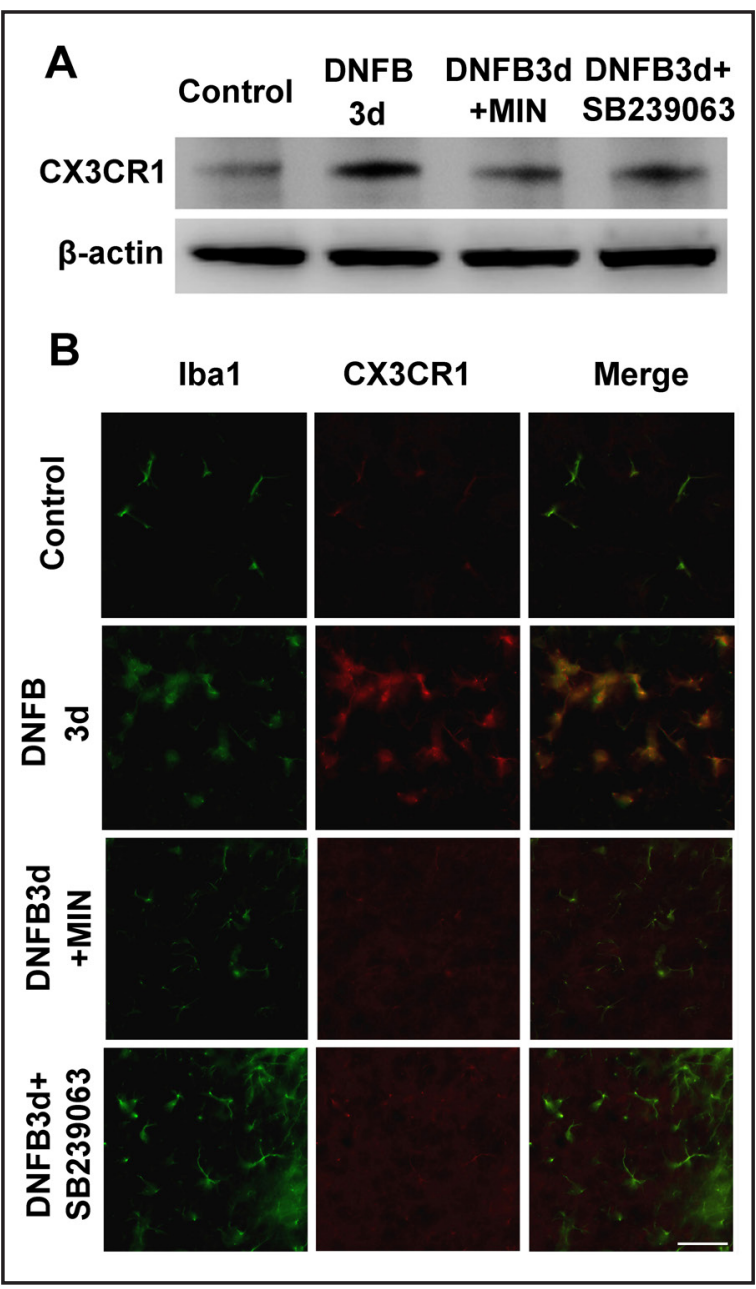
that the phosphorylation and activation of p38 is associated with the development of pruritus. Minocycline reduced activation of microglial cells as indicated by Iba1 staining, but SB239063 had little effect (Fig. 2A), suggesting that microglial activation promotes p38 phosphorylation but that $\mathrm{p} 38$ is not required for microglial activation.

We also examined total and p-p38 levels by western blot (Fig. 2C). The level of p-p38 protein in cervical spinal cord increased in animals receiving DNFB treatment, compared to control animals, while total p38 levels remained constant. Intrathecal administration of minocycline during DNFB treatment reduced p-p38 levels compared to mice that did not receive the drug, but p-p38 levels still exceeded those of control animals not painted with DNFB. In contrast, administration SB239063 resulted in a more extensive reduction in p-p38 expression $3 \mathrm{~d}$ after the end of DNFB treatment to levels seen in control non-painted mice. The decrease in p-p38 expression with SB239063 was associated with significantly diminished scratching behavior $3 \mathrm{~d}$ after cessation of DNFB treatment (Fig. 2D), but scratching did not significantly differ between the treatment groups at the $7 \mathrm{~d}$ time point. These results suggest that p38 phosphorylation in activated spinal microglial cells is involved in the development of pruritus in response to DNFB treatment.

CX3CR1 expression in microglia was induced by DNFB treatment

We hypothesized that microglia may mediate the development of chronic pruritus via mechanisms similar to those regulating chronic pain, so we examined the role of FKN/ CX3CR1 signaling in our mouse model of chronic pruritus. Expression of the FKN receptor CX3CR1 was first analyzed by western blot in control animals receiving no treatment, in animals treated with DNFB alone, and in animals treated with DNFB and concomitantly 


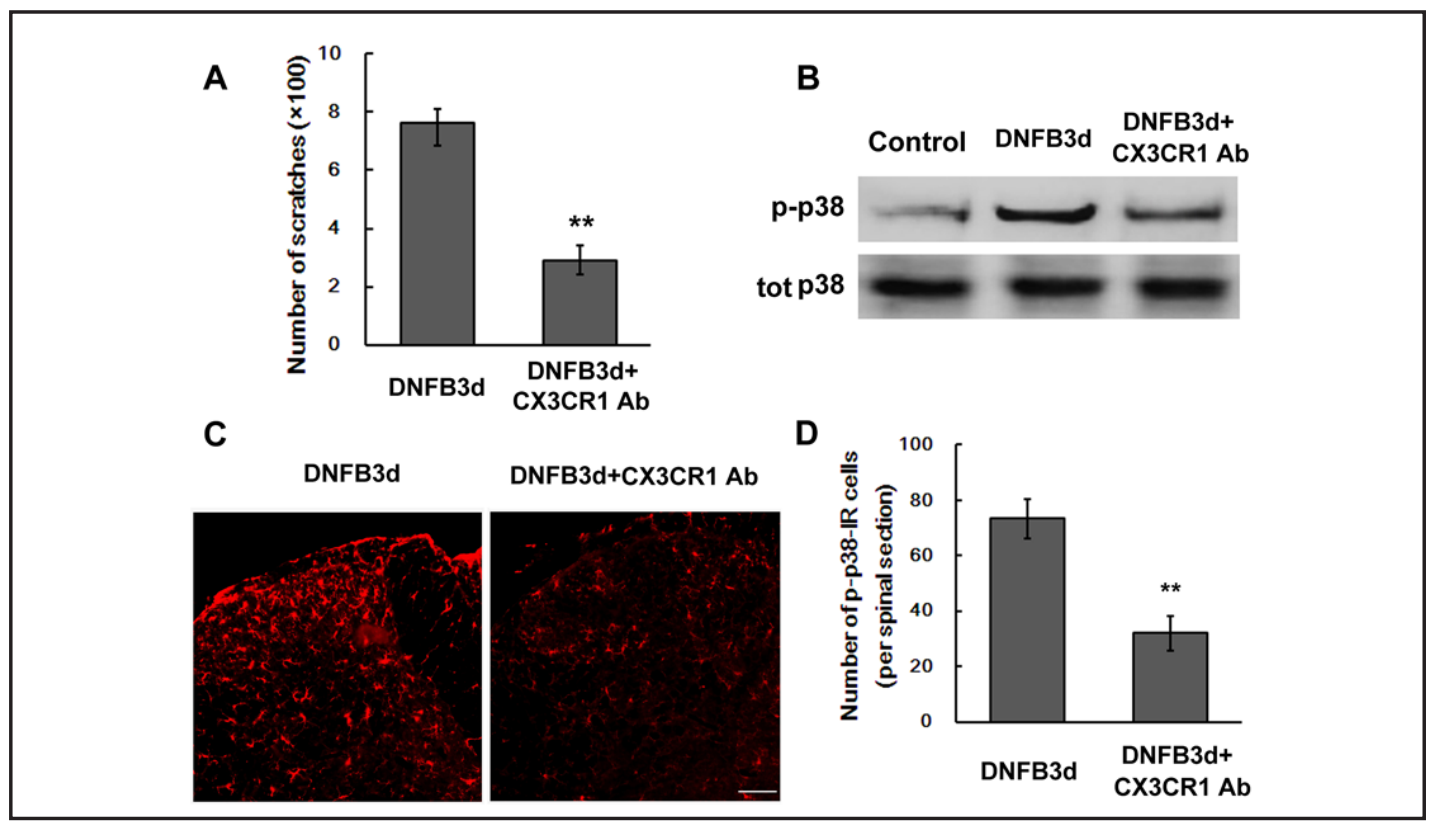

Fig. 4. DNFB-induced p38 activation was inhibited by CX3CR1 antibody. (A)Bar graph showing the number of hind paw scratches recorded in 30 min $3 \mathrm{~d}$ after final DNFB treatment in animals treated with DNFB alone and in animals receiving DNFB with intrathecal injections of anti-CX3CR1 antiserum. ${ }^{* *} \mathrm{P}<0.01$; bars represent mean \pm SEM; $n=6$ for each group. (B) Representative western blot of p-p38 and total p38 expression in spinal cord extracts $3 \mathrm{~d}$ after final DNFB painting from animals receiving control acetone paintings, DNFB treatment only, and DNFB treatment with intrathecal administration of anti-CX3CR1 antiserum. (C) Representative image showing p-p38 immunostaining in spinal cord sections from animals treated with DNFB only and DNFB with intrathecal injections of anti-CX3CR1 antiserum. Scale bar equals $50 \mu \mathrm{m}$. (D) Bar graph showing the number of p-p38 IR cells per spinal cord section from animals with treatments indicated in (A). ${ }^{* *} \mathrm{P}<0.01$; bars represent mean \pm SEM; $n=6$ for each group; Six sections per animal were selected for counting.

with minocycline or SB239063. Compared to control animals, CX3CR1expression greatly increased $3 \mathrm{~d}$ following DNFB treatment and diminished slightly in animals receiving either minocycline or SB239063 (Fig. 3A). Immunohistochemistry showed a similar pattern of expression for CX3CR1 (Fig. 3B), with little expression in control animals, greatly enhanced staining in animals $3 \mathrm{~d}$ after the last DNFB treatment, and reduced expression in animals treated with either minocycline or SB239063. minocycline reduced both microglial activation and CX3CR1 expression, but SB239063 had no effect on microglial activation, corroborating previous results. Thus, the mechanism mediating microglial activation appears to be distinct from the p38 MAPK signaling pathway regulating CX3CR1 expression.

FKN/CX3CR1 signaling was involved in the activation of p38 and the development of chronic pruritus.

The increase in CX3CR1 expression following DNFB treatment suggested a role for FKN signaling in the development of chronic pruritus. To examine this putative signaling pathway, we administered daily intrathecal injections of a neutralizing antibody against the CX3CR1 receptor to block FKN signaling during DNFB painting, then assessed activation of the downstream p38 MAPK signaling pathway. Administration of the antibody reduced scratching behavior $3 \mathrm{~d}$ after the final DNFB treatment (Fig. 4A) and decreased the level of p-p38 in spinal cord homogenates as shown by western blot (Fig. 4B). Total p38 expression was unchanged by DNFB or CX3CR1 antibody. The decrease in p-p38 expression by antibody administration is reflected in diminished p-p38 staining in cervical spinal cord sections by immunohistochemistry (Fig. 4C). Quantitation of immunoreactive microglial cells showed a 


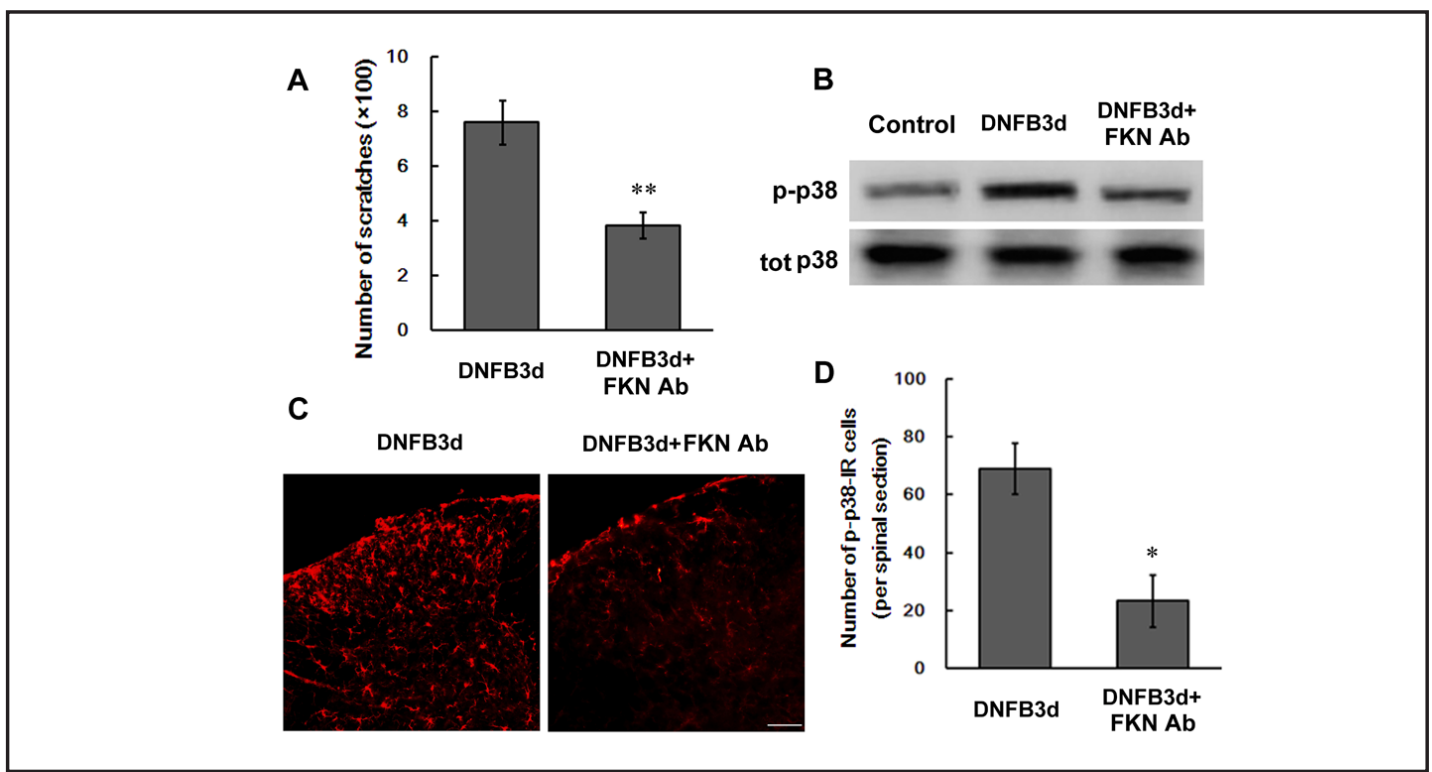

Fig. 5. DNFB-induced p38 activation was inhibited by FKN antibody. (A) Bar graph showing the number of hind paw scratches recorded in 30 min $3 \mathrm{~d}$ after final DNFB treatment in animals treated with DNFB alone and in animals receiving DNFB with intrathecal injections of anti-FKN antiserum. ${ }^{* *} \mathrm{P}<0.01$; bars represent mean \pm SEM; $n=6$ for each group. (B) Representative western blot of p-p38 and total p38 expression in spinal cord extracts $3 \mathrm{~d}$ after final DNFB painting from animals receiving control acetone paintings, DNFB treatment only, and DNFB treatment with concomitant administration of anti-FKN antiserum. (C) Representative image showing p-p38 immunostaining in spinal cord sections from animals treated with DNFB only and DNFB with intrathecal injections of anti-FKN antiserum. Scale bar equals $50 \mu \mathrm{m}$. (D) Bar graph showing the number of p-p38 IR cells per spinal cord section from animals with treatments indicated in (A). ${ }^{* * P}<0.01$; bars represent mean \pm SEM; $n=6$ for each group; Six sections per animal were randomly selected for counting.

significant $50 \%$ reduction in p-p38-labeled cells in the spinal cords of animals treated with the antibody during DNFB painting compared to animals receiving DNFB treatment alone (Fig. 4D). Similar results were obtained when a polyclonal FKN antibody was substituted for anti-CX3CR1 (Fig. 5). Scratching behavior was reduced (Fig. 5A), phosphorylation of p38 was inhibited (Fig. 5B), and immunohistochemical visualization of p-p38 was diminished (Fig. 5C) as was the number of p-p38-immunoreactive cells quantified per spinal section (Fig. 5D). These results suggest that development of chronic pruritus in our DNFB model is mediated by FKN signaling through the CX3CR1 receptor and downstream activation of the p38 MAPK pathway.

\section{Discussion}

There is increasing evidence supporting a role for microglia in the pathogenesis of pain. Because pain and itch are closely related somatosensory sensations processed by secondorder neurons in the dorsal root ganglion [30], microglial cells may act as neuroimmune regulators of both sensations [20]. In our study, we examined the potential role of microglia as mediators of chronic pruritus in a mouse model using repeated applications of DNFB to produce prolonged scratching for at least $7 \mathrm{~d}$ beyond the last pruritogenic application. Pruritus was associated with activation of spinal microglia monitored by increased expression of the microglial marker Iba1 and increased phosphorylation and activation of p38 MAPK. Intrathecal injections of microglial and p38 inhibitors during DNFB treatment reduced scratching and microglial activation. Chronic itch in our model was also associated with 
increased expression of the FKN receptor CX3CR1, and expression of CX3CR1 was inhibited by either microglial or p38 inhibitors. Activation of p38 was diminished by intrathecal injections of either anti-CX3CR1 or anti-FKN antibodies. We conclude that chronic pruritus in our model is mediated by spinal microglial activation via increased FKN signaling through the CX3CR1 receptor utilizing downstream p38 MAPK intracellular pathways.

It was interesting to note that increased scratching in our DNFB model occurred as early as $2 \mathrm{~h}$ after the final application of DNFB, whereas microglial activation, monitored by expression of Iba1, did not become apparent until $3 \mathrm{~d}$ after the final DNFB treatment. Scratching behavior at the $2 \mathrm{~h}$ time point may possibly reflect the response to acute pruritus mediated primarily by histamine-responsive neurons [8]. In contrast, chronic pruritus persisting after withdrawal of the pruritogenic stimulus may utilize mechanisms that activate microglia at a later time point. The failure of minocycline, which specifically inhibits microglial activation, to reduce scratching at the $2 \mathrm{~h}$ time point is a further indication that microglia may not mediate acute itch.

Despite the association of pain and pruritus, there are still differences in the pathways which mediate each sensation. For example, following spinal nerve ligation, Jin et al. reported that the level of p-p38 localized to spinal microglia increased after $0.5 \mathrm{~d}$ followed by delayed activation dorsal root ganglia neurons [26]. In contrast, in our model of chronic pruritus, we observed little change in p38 activation $2 \mathrm{~h}$ after the final DNFB painting, but expression of p-p38 was maximal $3 \mathrm{~d}$ later. Thus, downstream signaling may differ in neuropathic pain and involve earlier microglial p38 activation followed by later co-activation of p38 in both neurons and microglia.

The p38 enzymes represents a group of enzymes in the MAPK family that are phosphorylated and activated by a variety of physical and chemical stimuli [40]. In vitro studies have shown that activated p38 MAPK is present in spinal microglia and participates in signaling pathways that mediate nociception at the spinal cord level $[41,42]$. However, the role of p38 in the development of chronic pruritus has not been elucidated. In our study, we examined total and phosphorylated p38 expression and showed increased levels of phosphorylated p38 by western blot $3 \mathrm{~d}$ and $7 \mathrm{~d}$ after the final treatment with DNFB, while total p38 remained constant. The number of microglial cells immunopositive for p-p38 in cervical spinal cord sections was increased at the same time points, validating our western blot results. The increase in p-p38 levels appears to be a later event in our pruritus model since there was no change in p-p38 expression $2 \mathrm{~h}$ after the last DNFB treatment when Iba1 levels had already increased and scratching behavior was maximal. Thus, mechanisms involved in the induction of scratching behavior differ from those involved in prolonged chronic scratching. Nonetheless, minocycline inhibition of microglial activation was associated with diminished p38 activation at the $3 \mathrm{~d}$ time point and administration of the p38 inhibitor SB239063 produced an even more profound reduction in p-p38, showing the specificity of p38-dependent signaling on inhibition of scratching behavior. Despite continual administration of SB239063 until the animals were sacrificed, scratching increased $7 \mathrm{~d}$ after the final DNFB painting and was no longer significantly different from scratching in animals receiving only DNFB treatment. This increase in scratching at $7 \mathrm{~d}$ also occurred in minocycline-treated mice and may reflect sensitization to the itch-inhibiting pathways with continued minocycline and SB239063 exposure. In future experiments, we intend to examine p38 expression and activation at $7 \mathrm{~d}$ and later time points to address this question.

The association of chronic pain and pruritus in adults has been demonstrated in epidemiological studies [11] and may indicate a common mediating mechanism. FKN signaling via the CX3CR1 receptor activates microglia in nerve injury [33] and could play a similar role in the development of chronic itch. We examined expression of CX3CR1 in our DNFB model of chronic pruritus and the effect of inhibitors of the FKN/CX3CR1 signaling pathway on scratching behavior and phosphorylation of p38. CX3CR1 expression increased on day 3 after the last application of DNFB in coordination with enhanced microglial activation indicated by Iba1 staining. Inhibition of microglia activation by minocycline or disruption of p38 signaling pathways by SB239063 both reduced receptor expression.

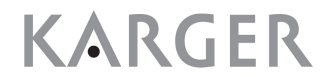


Intrathecal administration of antibodies against either CX3CR1 or FKN to disrupt signaling reduced p38 phosphorylation and suppressed scratching behavior. Thus, FKN/CX3CR1 signaling directly stimulated p38 activation in spinal microglia and may contribute to the development of chronic pruritus.

In conclusion, we have provided new evidence supporting a role for spinal microglial cells in the development of chronic pruritus in a mouse model induced by repeated applications of DNFB. The signaling pathways mediating pruritus appear similar to those implicated in the onset of neuropathic pain and likely involve FKN stimulation of CX3CR1 receptors leading to increased activation of the p38 MAPK pathway. We believe that future research focused on this pathway may lead to new clinical insights for improved treatment of chronic pruritus.

\section{Acknowledgments}

This work is supported by National Natural Science Foundation of China (81272083, 81301655) and Foundation of Shanghai Health Bureau (20124Y136).

\section{References}

1 Misery L, Brenaut E, Le Garrec R, Abasq C, Genestet S, Marcorelles P, Zagnoli F: Neuropathic pruritus. Nat Rev Neurol 2014;10:408-416.

2 Stander S, Weisshaar E, Mettang T, Szepietowski JC, Carstens E, Ikoma A, Bergasa NV, Gieler U, Misery L, Wallengren J, Darsow U, Streit M, Metze D, Luger TA, Greaves MW, Schmelz M, Yosipovitch G, Bernhard JD: Clinical classification of itch: A position paper of the international forum for the study of itch. Acta Derm Venereol 2007;87:291-294.

3 Kremer AE, Feramisco J, Reeh PW, Beuers U, Oude Elferink RP: Receptors, cells and circuits involved in pruritus of systemic disorders. Biochim Biophys Acta 2014;1842:869-892.

4 Oaklander AL: Neuropathic itch. Semin Cutan Med Surg 2011;30:87-92.

5 Dhand A, Aminoff MJ: The neurology of itch. Brain 2014;137:313-322.

6 Goodkin R, Wingard E, Bernhard JD: Brachioradial pruritus: Cervical spine disease and neurogenic/ neuropathic [corrected] pruritus. J Am Acad Dermatol 2003;48:521-524.

7 Ikoma A: Updated neurophysiology of itch. Biol Pharm Bull 2013;36:1235-1240.

-8 Schmelz M, Schmidt R, Bickel A, Handwerker HO, Torebjork HE: Specific c-receptors for itch in human skin. J Neurosci 1997;17:8003-8008.

-9 Bautista DM, Wilson SR, Hoon MA: Why we scratch an itch: the molecules, cells and circuits of itch. Nat Neurosci. 2014;17:175-182.

10 LaMotte RH, Dong X, Ringkamp M: Sensory neurons and circuits mediating itch. Nat Rev Neurosci 2014;15:19-31.

11 Dalgard F, Dawn AG, Yosipovitch G: Are itch and chronic pain associated in adults? Results of a large population survey in norway. Dermatology 2007;214:305-309.

12 Hachisuka J, Ross SE: Understanding the switch from pain-to-itch in dermatitis. Neurosci Lett 2014;579:188-189.

13 Akiyama T, Carstens E: Neural processing of itch. Neuroscience 2013;250:697-714.

14 Braz JM, Juarez-Salinas D, Ross SE, Basbaum AI: Transplant restoration of spinal cord inhibitory controls ameliorates neuropathic itch. J Clin Invest 2014;124:3612-3616.

15 Liu T, Gao YJ, Ji RR: Emerging role of toll-like receptors in the control of pain and itch. Neurosci Bull 2012;28:131-144.

16 Mochizuki H, Baumgartner U, Kamping S, Ruttorf M, Schad LR, Flor H, Kakigi R, Treede RD: Corticosubcortical activation patterns for itch and pain imagery. Pain 2013;154:1989-1998.

17 Pfab F, Valet M, Napadow V, Tolle TR, Behrendt H, Ring J, Darsow U: Itch and the brain. Chem Immunol Allergy 2012;98:253-265.

18 Weisshaar E, Szepietowski JC, Darsow U, Misery L, Wallengren J, Mettang T, Gieler U, Lotti T, Lambert J, Maisel P, Streit M, Greaves MW, Carmichael AJ, Tschachler E, Ring J, Stander S: European guideline on chronic pruritus. Acta Derm Venereol 2012;92:563-581. 
19 Ballantyne JC, Loach AB, Carr DB: Itching after epidural and spinal opiates. Pain 1988;33:149-160.

20 Smith HS: Activated microglia in nociception. Pain physician 2010;13:295-304.

21 DeLeo JA, Tanga FY, Tawfik VL: Neuroimmune activation and neuroinflammation in chronic pain and opioid tolerance/hyperalgesia. Neuroscientist 2004;10:40-52.

22 Hanisch, U.-K: Microglia as a source and target of cytokines. Glia 2002;40:140-155

23 Ito D, Imai Y, Ohsawa K, Nakajima K, Fukuuchi Y, Kohsaka S: Microglia-specific localisation of a novel calcium binding protein, iba1. Brain Res Mol Brain Res 1998;57:1-9.

24 McMahon, S. B, Cafferty, W. B. J, Marchand, F: Immune and glial cell factors as pain mediators and modulators. Exp Neurol. 2005;192:444-462

25 Zhuang ZY, Gerner P, Woolf CJ, Ji RR: ERK is sequentially activated in neurons, microglia, and astrocytes by spinal nerve ligation and contributes to mechanical allodynia in this neuropathic pain model. Pain 2005;114:149-159.

-26 Jin SX, Zhuang ZY, Woolf CJ, Ji RR:p38 mitogen-activated protein kinase is activated after a spinal nerve ligation in spinal cord microglia and dorsal root ganglion neurons and contributes to the generation of neuropathic pain. J Neurosci.2003;23:4017-4022.

27 Tikka, T, Fiebich, B. L, Goldsteins, G, Keinänen, R, and Koistinaho, J: Minocycline, a Tetracycline Derivative, Is Neuroprotective against Excitotoxicity by Inhibiting Activation and Proliferation of Microglia. J Neurosci. 2001;21:2580-2588.

28 Hains BC, Waxman SG: Activated microglia contribute to the maintenance of chronic pain after spinal cord injury. J Neurosci 2006;26:4308-4317.

29 Raghavendra V, Tanga F, Rutkowski MD, DeLeo JA: Anti-hyperalgesic and morphine-sparing actions of propentofylline following peripheral nerve injury in rats: Mechanistic implications of spinal glia and proinflammatory cytokines. Pain 2003;104:655-664.

-30 Zhang L, Jiang GY, Song NJ, Huang Y, Chen JY, Wang QX, Ding YQ: Extracellular signal-regulated kinase (ERK) activation is required for itch sensation in the spinal cord. Mol Brain 2014;7:25.

- 31 Tsuda M, Shigemoto-Mogami Y, Koizumi S, Mizokoshi A, Kohsaka S, Salter MW, Inoue K: P2x4 receptors induced in spinal microglia gate tactile allodynia after nerve injury. Nature 2003;424:778-783.

- 32 Verge GM, Milligan ED, Maier SF, Watkins LR, Naeve GS, Foster AC: Fractalkine (cx3cl1) and fractalkine receptor (cx3cr1) distribution in spinal cord and dorsal root ganglia under basal and neuropathic pain conditions. Eur J Neurosci 2004;20:1150-1160.

-33 Zhuang ZY, Kawasaki Y, Tan PH, Wen YR, Huang J, Ji RR: Role of the cx3cr1/p38 mapk pathway in spinal microglia for the development of neuropathic pain following nerve injury-induced cleavage of fractalkine. Brain Behav Immun 2007;21:642-651.

-34 Bazan JF, Bacon KB, Hardiman G, Wang W, Soo K, Rossi D, Greaves DR, Zlotnik A, Schall TJ: A new class of membrane-bound chemokine with a cx3c motif. Nature 1997;385:640-644.

-35 Milligan ED, Zapata V, Chacur M, Schoeniger D, Biedenkapp J, O’Connor K A, Verge GM, Chapman G, Green P, Foster AC, Naeve GS, Maier SF, Watkins L R: Evidence that exogenous and endogenous fractalkine can induce spinal nociceptive facilitation in rats. Eur J Neurosci.2004:20:2294-2302.

-36 Inagaki N, Shiraishi N, Igeta K, Itoh T, Chikumoto T, Nagao M, Kim JF, Nagai H: Inhibition of scratching behavior associated with allergic dermatitis in mice by tacrolimus, but not by dexamethasone. Eur J Pharmacol. 2006;546:189-196.

37 Shimada SG, LaMotte RH Behavioral differentiation between itch and pain in mouse. Pain 2008;139:681687.

-38 Sun YG, Chen ZF: A gastrin-releasing peptide receptor mediates the itch sensation in the spinal cord. Nature 2007;448:700-703.

39 Nojima H, Carstens E:5-Hydroxytryptamine (5-HT)2 Receptor Involvement in Acute 5-HT-Evoked Scratching but Not in Allergic Pruritus Induced by Dinitrofluorobenzene in Rats. J Pharmacol Exp Ther.2003;306:245-252.

40 Obata T, Brown GE, Yaffe MB: Map kinase pathways activated by stress: The p38 mapk pathway. Crit Care Med 2000;28:N67-77.

41 Ji RR, Suter MR: P38 mapk, microglial signaling, and neuropathic pain. Mol Pain 2007;3:33.

42 Svensson CI, Marsala M, Westerlund A, Calcutt NA, CampanaWM, Freshwater JD, Catalano R, Feng Y, Protter A A, Scott B, Yaksh TL: Activation of p38 mitogen-activated protein kinase in spinal microglia is a critical link in inflammation-induced spinal pain processing. J Neurochem.2003;86:1534-1544. 